\title{
Short-term combined exercise training improves cardiorespiratory fitness and autonomic modulation in cancer patients receiving adjuvant therapy
}

\author{
Cristiano Mostarda",2, Jurema Castro-Filha', Andréa Dias Reis', Mário Sevílio Jr', Carlos José Dias 1,2, Antonio Carlos Silva-Filho 1,2, \\ João Batista Silva Garcia', Maria do Desterro Nascimento', Hélio José Coelho-Junior', Bruno Rodrigues,**
}

'Physical Education Department, Universidade Federal do Maranhão (UFMA), São Luis, Brazil

Laboratory of Cardiovascular Adaptations to Exercise (LACORE), São Luis, Brazil

${ }^{3}$ Faculty of Physical Education, Universidade Estadual de Campinas (UNICAMP), Campinas, Brazil

The present study aimed to investigate the impact of a short-term exercise training (ET) on the cardiorespiratory fitness and autonomic modulation of women with breast cancer who were receiving adjuvant radiotherapy, chemotherapy or hormonotherapy. Eighteen women previously diagnosed with breast cancer receiving adjuvant radiotherapy, chemotherapy or hormone therapy were randomly allocated into breast cancer nonexercise $(\mathrm{BC})$ and exercise groups $(\mathrm{BC}+\mathrm{Ex})$. Moreover, nine healthy physically inactive volunteers were recruited to compose the noncancer control group (CG). The $\mathrm{BC}+\mathrm{Ex}$ group was underwent to a combined ET program, which was based on resistance, aerobic and flexibility exercises. ET was performed 3 times a week, on nonconsecutive days, for 4 weeks at the hospital room under the professional supervision. In turn, BC and CG remained without be engaged in physical exercise programs. Volunteers were evaluated regarding their cardio- respiratory fitness and autonomic modulation (i.e., time, frequency domains, and nonlinear [symbolic analysis]) before and after the end of the ET program. A priori, data indicate that women patients with breast cancer showed impaired exercise tolerance, as well as autonomic dysfunction in comparison with age-matched healthy control subjects. However, a 1-month combined ET program could reverse such impairments, so that after the intervention, BC+Ex and CG showing similar results in the cardiorespiratory test and heart rate variability analysis. In conclusion, data of the current study indicate that 1 month of ET is able to reverse impaired cardiorespiratory fitness and autonomic modulation in women with breast cancer receiving adjuvant therapy.

Keywords: Physical exercise, Combined exercise training, Breast cancer, Autonomic modulation, Heart rate variability

\section{INTRODUCTION}

Breast cancer is a worldwide public health concern, once a high prevalence of this condition is observed in several countries, regardless the economic condition (Guerra et al., 2005). However, the burden of breast cancer mortality seems to occur in low-income countries; where around $70 \%$ of the deaths occur (Lee et al., 2012). Moreover, there is a consensus indicating that postmenopause women with a family history of cancer, who underwent hormonal therapy, and demonstrate a sedentary lifestyle, associated with bad eating habits, smoking, elevated alcohol consumption, and use of contraceptives display a high risk for the development of breast cancer (Uzunlulu et al., 2016).

In the last decades, cancer patients have shown increased surviv$\mathrm{al}$, which is a product of the advances in cancer treatment. However, most times, radiological and pharmacological treatments are associated with unwanted side effects in different tissues, such as skeletal muscle weakness and atrophy, causing low cardiorespiratory fitness and, consequently, poor prognosis (Blair et al., 1996; Lira et al., 2014; Peel et al., 2014; Vainshelboim et al., 2017). Further-
*Corresponding author: Bruno Rodrigues (iD https://orcid.org/0000-0003-4246-4703 Faculty of Physical Education, Universidade Estadual de Campinas (UNICAMP), Avenida Érico Veríssimo, 701, Cidade Universitária Zeferino Vaz, Barão Geraldo. 13083-851, Campinas, SP, Brazil

Tel: +55-19-35216788, Fax: +55-19-35216788, E-mail: prof.brodrigues.com

Received: July 15, 2017 / Accepted: October 16, 2017
This is an Open Access article distributed under the terms of the Creative Commons Attribution Non-Commercial License (http://creativecommons.org/licenses/by-nc/4.0/) which permits unrestricted non-commercial use, distribution, and reproduction in any medium, provided the original work is properly cited. 
more, an increasing number of evidence have shown the potential cardiotoxic effects of chemotherapuetic agents (e.g., doxorubicin, anthracycline), including, but not limited to, alterations on cardiac function (i.e., left ventricular dysfunction) and autonomic modulation (Ades et al., 2014; Raschi et al., 2010).

In fact, short- and long-term exposing to antineoplastic agents have been described as a trigger factor to development of autonomic dysfunction, promoting direct effects on the cardiovascular system, such as increased heart rate, and impaired atrioventricular node conduction and cardiac chronotropism (Chuang et al., 2011; Lakoski et al., 2015; Nousiainen et al., 2001). Moreover, the autonomic system is responsible for the functioning of the hypothalamic pituitary-adrenal axis and renin-angiotensin aldosterone system and any disturbance in its function can promote increase on oxidative stress, inflammatory state, collaborating to atherosclerosis progression (Lakoski et al., 2015).

It should be stressed that cardiac autonomic dysfunction has been recognized as an important parameter to predict mortality in patients with cardiovascular diseases and breast cancer (de Seixas et al., 2010). Moreover, in addition to the cardiotoxic effects of chemotherapy, other factors such as sleep disturbances, psychosocial stress, metabolic disorders, and low physical capacity can be present in breast cancer patients. Each of these possible factors can coexist and contribute to autonomic dysfunction and cardiovascular risk (do Nascimento et al., 2012). Thus, emerging evidence has shown that heart rate and heart rate variability (HRV) parameters can predict functioning and survival among patients with metastatic and recurrent breast cancer (Battaglini et al., 2006).

On the other hand, exercise training (ET) is an important nonpharmacological tool in the oncology setting, once evidence indicate that exercise practice can promote substantial benefits in several clinical signals showed by cancer patients, such as cardiorespiratory fitness, muscle strength, fatigue, quality of life, and depressive symptoms, to name a few (De Backer et al., 2007). Despite the importance of this phenomenon, just a few studies have been addressed to investigate the effects of ET on the autonomic modulation of cancer patients (Riesenberg and Lübbe, 2010; Shin et al., 2016), mainly in cancer patients who were receiving adjuvant radiotherapy, chemotherapy and/or hormone therapy.

Therefore, the present study aimed at investigating the impact of a short-term ET on the cardiorespiratory fitness and autonomic modulation, evaluated by linear and nonlinear analysis, in women with breast cancer who were receiving adjuvant radiotherapy, chemotherapy or hormonotherapy. Moreover, data will be compared with an aged-matched healthy sedentary control group.

\section{MATERIALS AND METHODS}

The present investigation has a Randomized-Experimental design, which aimed to determine the effects of a 4-week supervised, hospital-based ET program in the cardiorespiratory capacity and autonomic modulation of physically inactive breast cancer patients, as well as to compare the postintervention outcomes with a control group of volunteers who were not diagnosed with cancer. Therefore, subjects were underwent to evaluations of the cardiorespiratory capacity and autonomic modulation before and after 1 month.

\section{Subjects}

Eighteen women previously diagnosed with breast cancer (age, $30-59$ years) receiving adjuvant radiotherapy (38.9\%), chemotherapy $(27.8 \%)$, or hormone therapy $(33.3 \%)$ were recruited by convenience from the Aldenora Bello Cancer Hospital, Brazil to participate of the present study. In the current sample, $50 \%$ of the volunteers presented invasive ductal carcinoma, 38.8\% infiltrating ductal carcinoma, 5.6\% fusocellular neoplasm, and 5.6\% showed cancer in different sides.

Breast cancer patients were included in the present study based on the following inclusion criteria: (a) being currently treated with radiotherapy, chemotherapy, or hormone therapy; (b) oncological stage at levels I, II or III based on TNM (tumor, nearby lymph nodes, metastasized) system described by the National Cancer Institute; (c) have undergone treatment within the first 6 months after surgery; (d) physically inactive, characterized by less than 150 min of exercise per week; (e) normal left ventricle ejection fraction ( $>55 \%)$. All these clinical parameters were analyzed, recorded and controlled by specialized physicians (i.e., oncologists and cardiologists). The exclusion criteria were based on inability to move, pregnancy, leukopenia $(<5,000 / \mathrm{mL})$, clinical diagnosis of mental disorders (e.g., depression, anxiety), diabetes mellitus, hypertension, and cardiac morphological and/or functional alterations. After applying the inclusion and exclusion factors, we used a computer-generated list of random numbers to randomly allocate the patients into two groups: breast cancer nonexercised (BC) and exercised patients $(\mathrm{BC}+\mathrm{Ex})$.

The control group (CG) was formed by nine healthy physically inactive women (age, 30-59 years). Volunteers were not clinically diagnosed with cancer, cardiovascular (e.g., acute myocardial infarction, stroke, peripheral arterial disease, and transient ischemic disease), pulmonary, neurological, or psychiatric (e.g., Parkinson or Alzheimer disease), musculoskeletal, and metabolic disease (e.g., diabetes mellitus type II), comorbidities associated with 
greater risk of falls, as well as have not recent history of smoking or alcohol abuse. Volunteers did not receive diet or exercise recommendations; however, they were required not to change their diet or food habits and their activities of daily living during the entire study period.

All volunteers signed the informed consent form and completed all measurements. This study was approved by the Research Ethics Committee of the Federal University of Maranhão under protocol number 545547. This study was developed in accordance with the Declaration of Helsinki and according to Resolution 196/96 of the National Health Council.

\section{Evaluations}

All volunteers were instructed to refrain high-intensity physical activity for the $96 \mathrm{hr}$, as well as drinking alcoholic and caffeinated beverages during the $24 \mathrm{hr}$ before all evaluations. Baseline evaluations (i.e., before) were performed one week before the begin of the exercise program or at the corresponding time, as well as the final evaluations (i.e., after) were performed in the following week after the last session of exercise or at the corresponding time.

In addition to the primary outcomes of interest, which were analyzed through a cardiopulmonary submaximal test and HRV, hemodynamic parameters and body composition were also evaluated.

\section{Cardiorespiratory capacity}

Cardiorespiratory capacity was evaluated according to Neil et al. (2013) in a cycle ergometer (Ergo-Fit 167, ERGO-FIT GmbH \& Co., Pirmasens, Germany). A priori, after remained seated for $20 \mathrm{~min}$, volunteers were evaluated regarding their blood pressure (BP) (Cardiomed Plus model, CARDIOMED, Curitiba, Brazil) and heart (HR) (Polar FT2 model, Polar, Kempele, Finland). This evaluation also occurs every minute throughout the test and during a 3-min active recovery. The test started with a 5-min warm-up at a fixed load of $15 \mathrm{~W}$, followed by a $15-\mathrm{W}$ increment at each 3-min stage until voluntary exhaustion. Borg's scale 6-20 was recorded at the end of the protocol. $\mathrm{VO}_{2 \max }$ was determined according with the American College and Sports Medicine guidelines (Pollock et al., 1998), using the follow submaximal formula: $\mathrm{VO}_{2 \max }(\mathrm{mL} / \mathrm{kg} / \mathrm{min})=($ watts $/$ body weight $) \times 10.8+3.5$.

\section{Heart rate variability analysis}

HRV data was collected before and after the training regimen. The time series of heart rate (tachogram) consisting of beat-tobeat intervals were extracted from the 5-min iRR recordings. Ectopic beats and artifacts within the tachogram's were detected and replaced by interpolated adjacent beats by applying an adaptive filter to generate normal-to-normal (NN) interval time series. Two experienced evaluators filtered manually by visual inspection and then used an automatic filter through Kubios HRV (Biosignal Analysis and Medical Imaging Group, Finland, Kuopio, Finland) software.

The time domain measures of HRV that were computed included a mean value of $\mathrm{NN}$ interval time series (mean $\mathrm{NN}[\mathrm{ms}]$ ), the standard deviation of $\mathrm{NN}$ interval time series, the square root of the mean squared differences of successive NN intervals (RMS$\mathrm{SD})$. Frequency domain measures of HRV were characterized by using the fast Fourier transform (FFT), whereby the low-frequency $(0.04-0.15 \mathrm{~Hz})$, high frequency $(\mathrm{HF})(0.15-0.4 \mathrm{~Hz})$, and lowto-high-frequency ratio (LF/HF) components of the RR interval time series were measured.

The beat-by-beat data sets were converted to equidistant time series before applying the FFT to calculate the spectral powers. The Fourier LF and HF components were computed in normalized units (LFnu= LF/[LF+ HF] and HFnu = HF/[LF+HF]), which is the recommended analysis set forth by the task force for accurate assessment of autonomic modulation. The LF component is reported to reflect both sympathetic and vagal modulation, whereas the HF component appears to be the result of vagal modulation. Moreover, the LF/HF component has been proposed as a measure of the cardiac sympathovagal balance.

\section{Symbolic analysis}

A symbolic analysis was carried out according to the approach previously described and validated by Porta et al. (2007). For this method, the same five minutes of the iRR-selected recording was used. Then, a coarse graining approach based on a uniform quantization procedure was used to transform the iRR series into a sequence of symbols. The length was kept fixed in all analysis. The full range of the sequences was uniformly spread over six levels (from 0 to 5), and patterns of length were constructed. Therefore, each subject and each experimental condition had its range of $i R R$ intervals.

The Shannon entropy of the distribution of the patterns was calculated to provide a quantification of the complexity of the pattern distribution. All possible patterns (i.e., 216) were grouped without any loss into three families referred to as patterns with no variation. The sequences are spread on six levels, and all possible patterns are divided into four groups, consisting of patterns with (a) no variations ( $0 \mathrm{~V}$, three symbols equal, associated with sympathetic modulation); (b) one variation ( $1 \mathrm{~V}$, two symbols equal and 
one different associated with sympathetic and parasympathetic modulation); (c) two like variations (2LV and associated to parasympathetic modulation); and (d) two unlike variations (2UV and associated to parasympathetic modulation) (Guzzetti et al., 2005).

\section{Combined ET protocol}

Before the main period for ET, volunteers were subjected to an adaptation period. During this period, the exercise sessions were composed by resistance, aerobic and flexibility exercises and performed 3 times a week, with each session lasted approximately 70 $\min$.

The main period was based in a combined ET, which was composed of aerobic and resistance exercises performed in the same session. Exercise sessions were performed 3 times a week, on nonconsecutive days, for 4 weeks at the hospital room under the professional supervision of an experienced exercise physiologist. During the begin of each session, subjects performed $30 \mathrm{~min}$ of aerobic exercise at $60 \% \mathrm{VO}_{2 \max }$ in a cycle ergometer (Ergo-Fit 167). Immediately after this, participants performed a low to moderate resistance training. The program of resistance training was based on 3 sets of 8-12 submaximal repetitions of each exercise. Exercises were performed by alternating the major groups in the upper and lower extremities (alternating groups). The exercises that composed the adaptation period were: (a) squat, (b) shoulder press, (c) hip flexion, (d) barbell bent over row, and (e) French press. Dumbbells and TheraBands were used to promote adequate load, allowing the volunteers to reach the aimed resistance. Training load was adjusted by the rating of perceived exertion (RPE) method, using the adapted Borg scale (Day et al., 2004).

\section{Statistical analysis}

Normality of data was tested using the Kolmogorov-Smirnov test. A two-way analysis of variance followed by a Dunnett post hoc test were performed to identify differences among the different times of evaluations and treatments. Cohen effect size $d$ was calculated to assess the magnitude of the results. The effect size was classified according to Rhea, 2004 for untrained volunteers. The level of significance was $5 \%(P<0.05)$ and all procedures were performed using the GraphPad Prism 6.0. (GraphPad Software, San Diego, CA, USA). The intention-to-treat principle was applied to the analysis of the outcomes for all participants based on their assigned treatment.

\section{RESULTS}

Table 1 shows the cardiorespiratory capacity, as well as the morphological and hemodynamic characteristics (systolic [SBP] and diastolic blood pressure [DBP]) of the volunteers recruited in the current study before and after 4 weeks. As expected, BP measurements demonstrated a normotensive classification in all groups. Similarly, body mass index data were within the parameters of normality, and subjects were not classified as obese. No significant differences in morphological and hemodynamic parameters were observed between the groups before or after the intervention. Furthermore, it is important to mention that volunteer's attendance was $100 \%$ for all experimental sessions, and no adverse effects during or after the experimental sessions were observed.

Regarding the cardiorespiratory capacity, data indicated that both experimental groups- $\mathrm{BC}$ and $\mathrm{BC}+\mathrm{Ex}-$ showed a lower $\mathrm{VO}_{2 \max }$ in comparison with $\mathrm{CG}$ before and after 4 weeks. However, a significant $(P=0.0135)$ improvement was observed in $\mathrm{BC}+\mathrm{Ex}$ when the intragroup analysis was performed. When the results of the cardiorespiratory capacity were compared with reference values from the Brazilian population (Herdy et al., 2016), was possible observe that CG reached a good to excellent classificaition at baseline. On the other hand, $\mathrm{VO}_{2 \max }$ in cancer patients was classified as

Table 1. SBP, DBP, weight, height and BMI variables before and after 4 weeks of exercise training in woman with breast cancer

\begin{tabular}{|c|c|c|c|c|c|c|}
\hline \multirow{2}{*}{ Variable } & \multicolumn{3}{|c|}{ Before } & \multicolumn{3}{|c|}{ After } \\
\hline & Healthy control $(n=9)$ & $\mathrm{BC}(\mathrm{n}=9)$ & $B C+\operatorname{Ex}(n=9)$ & Healthy control $(n=9)$ & $\mathrm{BC}(\mathrm{n}=9)$ & $\mathrm{BC}+\mathrm{Ex}(\mathrm{n}=9)$ \\
\hline $\mathrm{SBP}(\mathrm{mmHg})$ & $110 \pm 9$ & $111 \pm 10$ & $117 \pm 16$ & $111 \pm 10$ & $109 \pm 8$ & $110 \pm 13$ \\
\hline $\mathrm{DBP}(\mathrm{mmHg})$ & $76 \pm 8$ & $71 \pm 10$ & $72 \pm 7$ & $78 \pm 9$ & $70 \pm 5$ & $71 \pm 7$ \\
\hline Body mass (kg) & $66 \pm 11$ & $64 \pm 12$ & $60 \pm 9$ & $66 \pm 11$ & $64 \pm 11$ & $60 \pm 5$ \\
\hline Height (cm) & $157 \pm 3$ & $156 \pm 5$ & $155 \pm 0.05$ & $157 \pm 3$ & $156 \pm 4$ & $155 \pm 0.05$ \\
\hline $\mathrm{BMI}\left(\mathrm{kg} / \mathrm{m}^{2}\right)$ & $26 \pm 3$ & $26 \pm 2$ & $25 \pm 3$ & $26 \pm 3$ & $26 \pm 2$ & $25 \pm 3$ \\
\hline $\mathrm{VO}_{2 \max }(\mathrm{mL} / \mathrm{kg} / \mathrm{min})$ & $36 \pm 6$ & $14 \pm 3^{*}$ & $16 \pm 2^{*}$ & $36 \pm 6$ & $15 \pm 3^{*}$ & $19 \pm 2^{*}$ \\
\hline
\end{tabular}

Values are presented as mean \pm standard deviation.

SBP, systolic blood pressure; DBP, diastolic blood pressure; BMI, body mass index; BC, breast cancer; Ex, exercise.

${ }^{*} P<0.05$ vs. control group. 
very weak to weak. After ET, values in the $\mathrm{BC}+\mathrm{Ex}$ were all classified as weak, indicating an important improvement (Herdy et al., 2016). ES classification corroborates with the hypothesis test and indicate that $\mathrm{BC}+$ Ex presents a moderate classification, whereas $\mathrm{BC}$ just showed a trivial classification (Table 2).

Table 2. Effect size and its classification

\begin{tabular}{|c|c|c|c|}
\hline Variable & Healthy control & $\mathrm{BC}$ & $B C+E x$ \\
\hline \multicolumn{4}{|l|}{ Cardiorespiratory fitness } \\
\hline $\mathrm{VO}_{2 \max }(\mathrm{mL} / \mathrm{kg} / \mathrm{min})$ & 0 (trivial) & 0.33 (trivial) & 1.5 (moderate) \\
\hline \multicolumn{4}{|l|}{ Time domain } \\
\hline $\mathrm{RR}(\mathrm{ms})$ & 1.69 (moderate) & 0.09 (trivial) & 0.67 (small) \\
\hline SDNN (ms) & 0.91 (small) & 0.23 (trivial) & 0.92 (small) \\
\hline RMSSD (ms) & 0.76 (small) & 0.27 (trivial) & 0.90 (small) \\
\hline \multicolumn{4}{|l|}{ Frequency domain } \\
\hline $\mathrm{LF}\left(\mathrm{ms}^{2}\right)$ & 2.06 (large) & 0.39 (trivial) & 0.59 (small) \\
\hline $\mathrm{HF}\left(\mathrm{ms}^{2}\right)$ & 0.07 (trivial) & 0.37 (trivial) & 5.51 (large) \\
\hline $\mathrm{LF}(\mathrm{nu})$ & 0.32 (trivial) & 0.28 (trivial) & 1.58 (moderate) \\
\hline$H F(n u)$ & 0.41 (trivial) & 0.22 (trivial) & 1.58 (moderate) \\
\hline $\mathrm{LF} / \mathrm{HF}$ & 0.45 (trivial) & 0.36 (trivial) & 1.31 (moderate) \\
\hline \multicolumn{4}{|l|}{ Symbolic analysis } \\
\hline OV (\%) & 0.24 (trivial) & 0.48 (trivial) & 5.61 (large) \\
\hline $1 \mathrm{~V}(\%)$ & 0.85 (small) & 0.44 (trivial) & 0 (trivial) \\
\hline 2LV (\%) & 1.49 (moderate) & 0.33 (trivial) & 5.47 (large) \\
\hline 2UV (\%) & 0.34 (trivial) & 0.53 (trivial) & 5.45 (large) \\
\hline
\end{tabular}

$\mathrm{BC}$, breast cancer; Ex, exercise; RR, interval RR; SDNN, standard deviation of interval RR; RMSSD, root mean square of successive differences; LF, low-frequency component; HF, high-frequency component; LF/HF, sympathovagal balance; OV three symbols equal, associated with sympathetic modulation; $1 \mathrm{~V}$, two symbols equal and one different associated with sympathetic and parasympathetic modulation; 2LV, two like variations and associated to parasympathetic modulation; 2UV, two unlike variations and associated to parasympathetic modulation.
Table 3 shows HRV analysis before and after 4 weeks. Results of the time domain analysis indicated that the cancer groups, regardless ET, showed a lower SDNN, RMSSD, and VAR-RR than CG before and after the protocol. However, ET was effective to cause increase in all these variables after 4 weeks. Regarding the frequency domain, before $\mathrm{ET}, \mathrm{BC}$, and $\mathrm{BC}+\mathrm{Ex}$ groups demonstrated lower absolute (ms) LF and HF, followed by lower relative (nu) HF of the HRV than CG. On the other hand, relative LF was higher in the cancer groups, regardless ET, than in CG. Consequently, sympathovagal evaluation demonstrated elevated values in $\mathrm{BC}$ and $\mathrm{BC}+\mathrm{Ex}$ when compared with CG. ET caused significant improvements in the autonomic balance, so that a marked increase on $\mathrm{HF}\left(\mathrm{ms}^{2}\right.$ and $\mathrm{nu}$ ) and decrease on LF (nu), eliciting significant decreasing on $\mathrm{LF} / \mathrm{HF}$, were observed in $\mathrm{BC}+\mathrm{Ex}$ in comparison with the baseline moment and $\mathrm{BC}$ group. Moreover, there were no more significant differences with the CG. In turn, time domain analysis in $\mathrm{BC}$ demonstrated a similar behavior with the observed 4 weeks before, and variables were different in comparison with the CG.

In relation to ES and its classification in the time domain, $\mathrm{BC}+\mathrm{Ex}$ demonstrates a higher classification (i.e., small) in all variables (i.e., RR, SDNN, RMSSD) in comparison with BC (Table 2). Equivalent results were observed in the frequency domain analyses, once just a trivial classification was reached by the $\mathrm{BC}$ group, whereas small (i.e., LF), moderate (i.e., LF [nu], HF [nu] and $\mathrm{LF} / \mathrm{HF})$ and large $\left(\mathrm{HF}\left[\mathrm{ms}^{2}\right]\right)$ classifications were observed in the $\mathrm{BC}+$ Ex group (Table 3).

The symbolic analysis indicated that the cancer groups showed a higher $0 \mathrm{~V} \%$, an index of sympathetic modulation, before four

Table 3. Autonomic variables in the time and frequency domains before and after 4 weeks of exercise training

\begin{tabular}{|c|c|c|c|c|c|c|}
\hline \multirow{2}{*}{ Variable } & \multicolumn{3}{|c|}{ Before } & \multicolumn{3}{|c|}{ After } \\
\hline & Control $(n=9)$ & $\mathrm{BC}(\mathrm{n}=9)$ & $B C+E x(n=9)$ & Control $(n=9)$ & $\mathrm{BC}(\mathrm{n}=9)$ & $B C+E x(n=9)$ \\
\hline \multicolumn{7}{|l|}{ Time domain } \\
\hline $\mathrm{RR}(\mathrm{ms})$ & $1,044 \pm 30$ & $610 \pm 507$ & $601 \pm 34$ & $857 \pm 153$ & $651 \pm 350$ & $1,015 \pm 863$ \\
\hline SDNN (ms) & $66 \pm 10$ & $27 \pm 10^{*}$ & $20 \pm 15^{*}$ & $59 \pm 4$ & $25 \pm 7.2^{*}$ & $30 \pm 3.7^{*}$ \\
\hline $\mathrm{RMSSD}$ (ms) & $40 \pm 7$ & $15 \pm 9.4^{*}$ & $15 \pm 8^{*}$ & $48 \pm 13$ & $17 \pm 5.6^{*}$ & $27 \pm 17^{*}$ \\
\hline \multicolumn{7}{|c|}{ Frequency domain } \\
\hline $\operatorname{LF}\left(\mathrm{ms}^{2}\right)$ & $594 \pm 94$ & $149 \pm 128^{*}$ & $122 \pm 117^{*}$ & $834 \pm 135$ & $205 \pm 153$ & $550 \pm 1,004$ \\
\hline $\mathrm{HF}\left(\mathrm{ms}^{2}\right)$ & $781 \pm 130$ & $90 \pm 84^{*}$ & $73 \pm 92^{*}$ & $772 \pm 92$ & $119 \pm 69^{*}$ & $701 \pm 132^{\#}$ \\
\hline $\mathrm{LF}(\mathrm{nu})$ & $46 \pm 10$ & $65.2 \pm 12^{*}$ & $66 \pm 12^{*}$ & $50 \pm 14$ & $62 \pm 10^{*}$ & $47 \pm 12^{\#,+}$ \\
\hline$H F(n u)$ & $45 \pm 10$ & $34.4 \pm 12^{*}$ & $34 \pm 12^{*}$ & $50 \pm 14$ & $37 \pm 11^{*}$ & $53 \pm 12^{\#, \dagger}$ \\
\hline $\mathrm{LF} / \mathrm{HF}$ & $0.91 \pm 0.4$ & $2.3 \pm 1.2^{*}$ & $2.3 \pm 1.3^{*}$ & $1.17 \pm 0.7$ & $1.9 \pm 1$ & $1 \pm 0.5^{\#}$ \\
\hline
\end{tabular}

$\mathrm{BC}$, breast cancer; Ex, exercise; RR, interval RR; SDNN, standard deviation of interval RR; RMSSD, root mean square of successive differences; LF, low-frequency component, $H F$, high-frequency component; LF/HF, sympathovagal balance.

${ }^{*} P<0.05$ vs. control. ${ }^{\#} P<0.05$ vs. BC+Ex before 4 weeks. ${ }^{\dagger} P<0.05 \mathrm{BC}$ after 4 weeks. 
weeks of ET than the CG (Table 4, Fig. 1). ET could decrease 0V\% after four weeks compared to the pre-exercise values. However, after 4 weeks, values still elevated in the BC, while a signifi- cant decrease were observed in the trained group. Moreover, values in the $\mathrm{BC}+$ Ex were similar with the age-matched control group. Regarding the vagal modulation, which was evaluated by the $2 \mathrm{LV}$

Table 4. Autonomic variables in the symbolic analysis before and after 4 weeks of exercise training

\begin{tabular}{|c|c|c|c|c|c|c|}
\hline \multirow{2}{*}{ Symbolic analysis } & \multicolumn{3}{|c|}{ Before } & \multicolumn{3}{|c|}{ After } \\
\hline & Control & $\mathrm{BC}$ & $\mathrm{BC}+\mathrm{Ex}$ & Control & $\mathrm{BC}$ & $\mathrm{BC}+\mathrm{Ex}$ \\
\hline $\mathrm{OV}(\%)$ & $18 \pm 9.0$ & $35 \pm 2.5^{*}$ & $36 \pm 3.0^{*}$ & $20 \pm 7.0$ & $34 \pm 1.5^{*}$ & $21 \pm 2.3^{\#, \dagger}$ \\
\hline 1V (\%) & $49 \pm 1.5$ & $49 \pm 2.0$ & $47 \pm 1.8$ & $46 \pm 4.7$ & $50 \pm 2.5$ & $47 \pm 1.5$ \\
\hline $2 \mathrm{LV}(\%)$ & $15 \pm 1.6$ & $5.2 \pm 0.8^{*}$ & $4.1 \pm 0.6^{*}$ & $13 \pm 5.0$ & $4.9 \pm 1.0^{*}$ & $10 \pm 1.4^{* * \#, t}$ \\
\hline $2 U V(\%)$ & $18 \pm 1.5$ & $11 \pm 1.7^{*}$ & $12 \pm 1.2^{*}$ & $20 \pm 8.0$ & $10 \pm 2.0^{*}$ & $21 \pm 2.0^{* .+}$ \\
\hline
\end{tabular}

Mann-Whitney test.

$\mathrm{BC}$, breast cancer; Ex, exercise; OV, three symbols equal, associated with sympathetic modulation); 1V, two symbols equal and one different associated with sympathetic and parasympathetic modulation; 2LV, two like variations and associated to parasympathetic modulation; 2UV, two unlike variations and associated to parasympathetic modulation.

${ }^{*} P<0.05$ vs. control. ${ }^{\#} P<0.05$ vs. BC+Ex before 4 weeks. ${ }^{\dagger} P<0.05$ vs. BC after 4 weeks.

OV

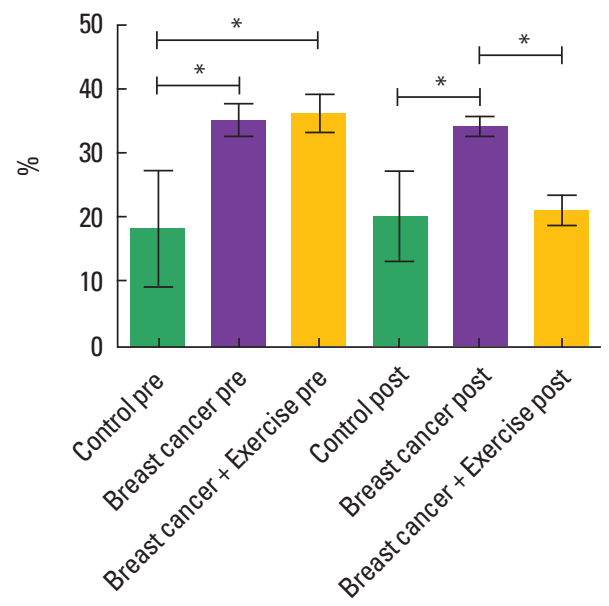

2LV

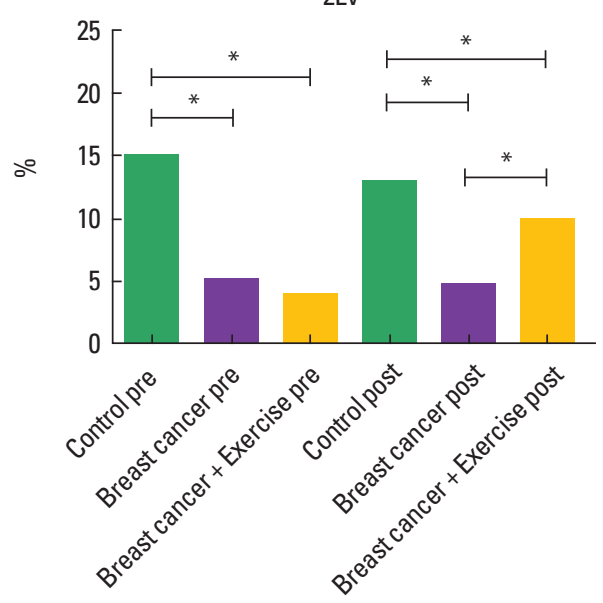

$1 \mathrm{~V}$

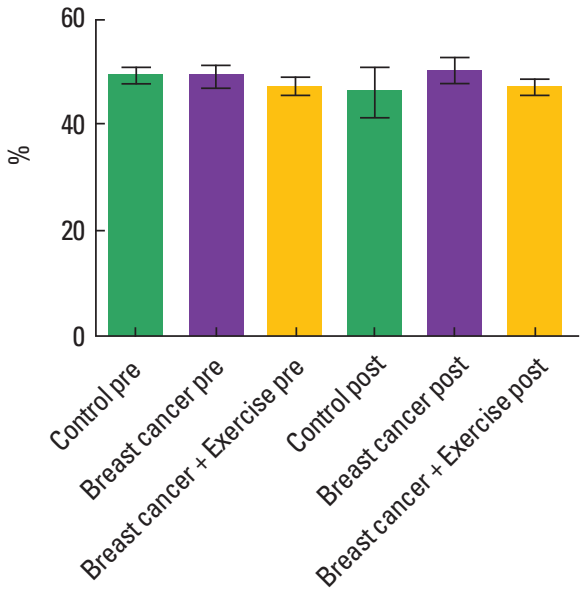

2UV

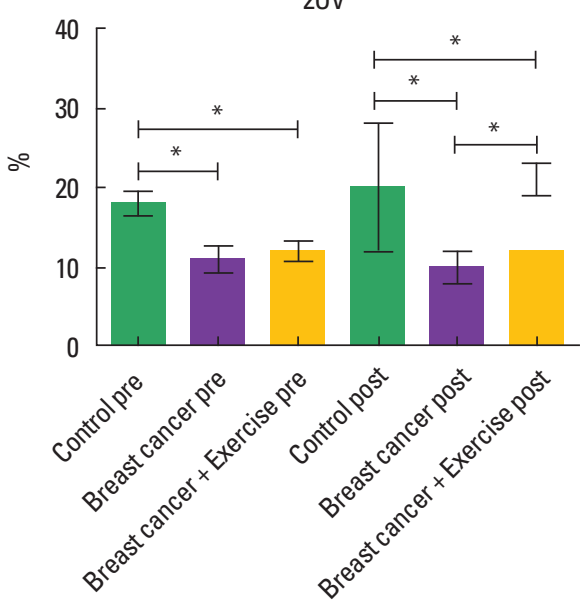

Fig. 1. Symbolic analysis graph trends before and after the ET. BC, breast cancer; Ex, exercise; OV, three symbols equal, associated with sympathetic modulation; 1V, two symbols equal and one different associated with sympathetic and parasympathetic modulation; 2LV, two like variations and associated to parasympathetic modulation; $2 \mathrm{UV}$, two unlike variations and associated to parasympathetic modulation. ${ }^{*} P<0.05$. 
and $2 \mathrm{UV}$, baseline evaluations demonstrated that $\mathrm{BC}$ and $\mathrm{BC}+\mathrm{Ex}$ showed lower results than CG. However, these components increased after 4 weeks of $\mathrm{ET}$ in $\mathrm{BC}+\mathrm{Ex}$ in relation to Baseline and BC. Nevertheless, after 4 weeks, differences were still significant among cancer groups-BC and $\mathrm{BC}+\mathrm{Ex}-$ and $\mathrm{CG}$ (Table 4, Fig. 1). In relation to $\mathrm{ES}$ classification, again, data corroborated with hypothesis test since $0 \mathrm{~V}(\%), 2 \mathrm{LV}(\%)$, and $2 \mathrm{UV}(\%)$ were classified as large and trivial in the $\mathrm{BC}+\mathrm{Ex}$ and $\mathrm{BC}$ groups, respectively (Table 2).

\section{DISCUSSION}

The main findings of the current study indicate that women patients with breast cancer show impaired exercise tolerance and autonomic dysfunction in comparison with age-matched healthy control subjects. However, a 1-month combined ET program is able to reverse such impairments, so that after the intervention, $\mathrm{BC}+\mathrm{Ex}$ and $\mathrm{CG}$ showing similar results in the cardiorespiratory test and HRV analysis (i.e., time, frequency domains, and nonlinear [symbolic analysis]).

Several authors have shown the importance of cardiorespiratory capacity evaluation in the monitoring and prognosis of cancer patients, once low $\mathrm{VO}_{2 \max }$ levels are strongly associated with increased risk for cardiovascular events, which are the leading cause of long-term mortality and morbidity in cancer survivors (Blair et al., 1996; Peel et al., 2014; Sanchis-Gomar et al., 2015; Vainshelboim et al., 2017). It is important to mention, that the pharmacological anticancer therapy has a key role in this phenomenon because many of the changes presented in cardiorespiratory capacity during cancer are associated with the pharmacological treatment (Møller et al., 2015).

On the other hand, physical exercise seems to be the only and most effectiveness therapy able to increase this variable in healthy and unhealthy patients (Sanchis-Gomar et al., 2015; Vainshelboim et al., 2017). However, results are controversial regarding the effects of ET on the cardiorespiratory capacity of cancer patients. In fact, some evidence are in line with the present study, demonstrating improved cardiorespiratory capacity in cancer patients after ET (Casla et al., 2015; Schmitt et al., 2016), whereas other studies refuted this hypothesis, and data indicate decreased $\mathrm{VO}_{2 \mathrm{pe}-}$ ak in trained cancer patients who were received adjuvant chemotherapy during the protocol of ET (Møller et al., 2015).

The inconsistencies in these findings could be a function of the differences in the sample characteristics, type of cancer, pharmacological therapy, as well as exercise prescription (e.g., intensity, vol- ume, frequency). Moreover, some studies have not only studied the effects of ET per se, and some protocols have aggregated ET and dietary counseling.

However, it is noteworthy that in, that in the study of MØller et al. (2015), for example, 12 weeks of combined ET_composed by high intensity resistance and interval aerobic training - was not able to avoid the significant decrease observed in the cardiorespiratory fitness $(-2.1 \mathrm{~mL} / \mathrm{min} / \mathrm{kg})$ of cancer patients. Therefore, in conjunction with the current data, is possible infer that the volume of aerobic training has an important role in the cardiorespiratory adaptations elicited by ET than exercise intensity and frequency, as well as the length of the exercise program. Nevertheless, future studies should be addressed to confirm this hypothesis.

Regarding autonomic modulation, data of the present study corroborate with several evidence in the literature (Adams et al., 2015; Palma et al., 2016; Vigo et al., 2015), once cancer patients demonstrated marked autonomic dysfunction at baseline in comparison with age-matched free-cancer controls (i.e., CG). Indeed, this phenomenon has been observed not only in patients diagnosed with breast cancer, but also in other kinds of cancer, such as gastrointestinal, pancreatic, colon (Adams et al., 2015; Palma et al., 2016; Vigo et al., 2015). In the experiment of Vigo et al. (2015), for example, authors demonstrated that breast cancer survivors showed lower HRV, HF band of HRV, and baroreflex sensitivity than healthy controls.

Moreover, our data indicate that a short-term program of combined ET is able to reverse cancer-induced autonomic dysfunction, so that a decreased sympathetic modulation-trough time domain, LF (nu) and sympathovagal balance (LF/HF), and symbolic measures, normalized component in $0 \mathrm{~V}(\%)$-as well as increased vagal modulation, which was also demonstrated by time domain, HF (nu) RMSSD indexes, and symbolic measures, 2LV (\%) and 2UV(\%) were observed in the trained cancer patients.

Just a few studies have investigated the impact of a combined ET programs in the HRV of cancer patients. Similar with the present study, Shin et al. (2016) recorded the impact of a shortterm ET program (8 weeks) in the autonomic modulation of breast cancer patients. Despite the lack of significant results in the intragroup analysis, results demonstrated that after 8 weeks of ET trained breast cancer patients showed higher results on HF, SDNN and RMSSD than nontrained breast cancer patients. These data are in line with the findings of Riesenberg and Lübbe (2010), which observed increased parasympathetic activity through RMSSD index in cancer patients underwent to a multidisciplinary rehabilitation program. 
Taken together, these data indicate that $\mathrm{ET}$ is able to reverse the deleterious effects of cancer, and probably cancer treatment, on autonomic modulation. However, it is noteworthy that the present study presents some characteristics that should be highlighted, such as a marked short-term of intervention, and an exercise program developed during cancer treatment. These features seem to be important, once cancer treatment is strongly associated with fatigue, nausea, vomiting, which can make the patient feel unmotivated, limiting exercise adherence (Møller et al., 2015). Therefore, a short-term ET program that demonstrates effectiveness to increase some parameters associated with quality of life and autonomy, such as cardiorespiratory fitness, may make patients feel excited and stimulated to participate of the ET program.

Moreover, there is no consensus about the practice of physical exercise during anticancer therapy, and some patients might not be able to perform the sessions of exercise due muscle weakness, fatigue and malaise (Rock et al., 2012). On the other hand, data of the present study demonstrate that the current combined ET program was well supported by breast cancer patients who were receiving adjuvant radiotherapy, chemotherapy and/or hormone therapy.

Some limitations of the present study should be mentioned to collaborate with better inferences about the data, such as the lack of evaluations of other important parameters that could be modulated by the present ET protocol (e.g., muscle strength, quality of live), and a long-term ET program. Thus, these limitations must be added in future studies that aimed to investigate the impact of a combined ET program in breast cancer patients.

In conclusion, data of the current study indicate that women patients with breast cancer show impaired exercise tolerance and autonomic dysfunction in comparison with age-matched healthy control subjects. However, a 1-month combined ET program is able to reverse such parameters to levels closely matched the presented by the aged-matched group.

\section{CONFLICT OF INTEREST}

No potential conflict of interest relevant to this article was reported.

\section{ACKNOWLEDGMENTS}

We would like to thanks Fundação de Amparo à Pesquisa do Maranhão - FAPEMA and Coordenação de Aperfeiçoamento de Pessoal do Ensino Superior - CAPES for scholarships. Also, we would like to thank Hospital Aldenora Bello - Fundação Antonio
Jorge Dino for the support for research. B.R. received financial support from Conselho Nacional de Pesquisa e Desenvolvimento (CNPq-BPQ).

\section{REFERENCES}

Adams SC, Schondorf R, Benoit J, Kilgour RD. Impact of cancer and chemotherapy on autonomic nervous system function and cardiovascular reactivity in young adults with cancer: a case-controlled feasibility study. BMC Cancer 2015;15:414.

Ades F, Zardavas D, Pinto AC, Criscitiello C, Aftimos P, de Azambuja E. Cardiotoxicity of systemic agents used in breast cancer. Breast 2014;23: 317-328.

Battaglini C, Bottaro M, Dennehy C, Barfoot D, Shields E, Kirk D, Hackney AC. Efeitos do treinamento de resistência na força muscular e níveis de fadiga em pacientes com câncer de mama. Rev Bras Med Esporte 2006;2:153-158.

Blair SN, Kampert JB, Kohl HW 3rd, Barlow CE, Macera CA, Paffenbarger RS Jr, Gibbons LW. Influences of cardiorespiratory fitness and other precursors on cardiovascular disease and all-cause mortality in men and women. JAMA 1996;276:205-210.

Casla S, Hojman P, Márquez-Rodas I, López-Tarruella S, Jerez Y, Barakat R, Martín M. Running away from side effects: physical exercise as a complementary intervention for breast cancer patients. Clin Transl Oncol 2015;17:180-196.

Chuang CY, Han WR, Li PC, Song MY, Young ST. Effect of long-term music therapy intervention on autonomic function in anthracycline-treated breast cancer patients. Integr Cancer Ther 2011;10:312316.

Day ML, McGuigan MR, Brice G, Foster C. Monitoring exercise intensity during resistance training using the session RPE scale. J Strength Cond Res 2004;18:353-358

De Backer IC, Van Breda E, Vreugdenhil A, Nijziel MR, Kester AD, Schep G. High-intensity strength training improves quality of life in cancer survivors. Acta Oncol 2007;46:1143-1151.

de Seixas RJ, Kessler A, Frison VB. Physical activity and quality of life in patients with cancer during chemotherapy treatment. Rev Bras Cancerol 2010;56:321-330.

do Nascimento EB, Leite RD, Prestes J. Cancer: benefits of resistence and aerobic training. Rev Educ Fís UEM 2012;22:651-658.

Guerra MR, Gallo CV, Mendonça GA, e Silva Mendonça GA. The risk of cancer in Brazil: tendencies and recent epidemiologic studies. Rev Bras Cancerol 2005;51:227-234.

Guzzetti S, Borroni E, Garbelli PE, Ceriani E, Della Bella P, Montano N, Cogliati C, Somers VK, Malliani A, Porta A. Symbolic dynamics of 
heart rate variability: a probe to investigate cardiac autonomic modulation. Circulation 2005;112:465-470.

Herdy AH, Caixeta A. Brazilian cardiorespiratory fitness classification based on maximum oxygen consumption. Arq Bras Cardiol 2016;106: 389-395.

Lakoski SG, Jones LW, Krone RJ, Stein PK, Scott JM. Autonomic dysfunction in early breast cancer: Incidence, clinical importance, and underlying mechanisms. Am Heart J 2015;170:231-241.

Lee BL, Liedke PE, Barrios CH, Simon SD, Finkelstein DM, Goss PE. Breast cancer in Brazil: present status and future goals. Lancet Oncol 2012;13:e95-102.

Lira FS, Neto JC, Seelaender M. Exercise training as treatment in cancer cachexia. Appl Physiol Nutr Metab 2014;39:679-686.

Møller T, Lillelund C, Andersen C, Bloomquist K, Christensen KB, Ejlertsen B, Nørgaard L, Wiedenbein L, Oturai P, Breitenstein U, Adamsen L. The challenge of preserving cardiorespiratory fitness in physically inactive patients with colon or breast cancer during adjuvant chemotherapy: a randomised feasibility study. BMJ Open Sport Exerc Med 2015;1:e000021.

Neil SE, Klika RJ, Garland SJ, McKenzie DC, Campbell KL. Cardiorespiratory and neuromuscular deconditioning in fatigued and non-fatigued breast cancer survivors. Support Care Cancer 2013;21:873-881.

Nousiainen T, Vanninen E, Jantunen E, Remes J, Ritanen E, Vuolteenaho $\mathrm{O}$, Hartikainen J. Neuroendocrine changes during the evolution of doxorubicin-induced left ventricular dysfunction in adult lymphoma patients. Clin Sci (Lond) 2001;101:601-607.

Palma MR, Vanderlei LC, Ribeiro FE, Mantovani AM, Christofaro DG, Fregonesi CE. The relationship between post-operative time and cardiac autonomic modulation in breast cancer survivors. Int J Cardiol 2016;224:360-365.

Peel AB, Thomas SM, Dittus K, Jones LW, Lakoski SG. Cardiorespiratory fitness in breast cancer patients: a call for normative values. J Am Heart Assoc 2014;3:e000432.

Pollock ML, Gaesser GA, Butcher JD, Després JP, Dishman RK, Franklin BA, Garber CE. ACSM position stand: the recommended quantity and quality of exercise for developing and maintaining cardiorespira- tory and muscular fitness, and flexibility in healthy adults. Med Sci Sports Exerc 1998;30:975-991.

Porta A, Tobaldini E, Guzzetti S, Furlan R, Montano N, Gnecchi-Ruscone T. Assessment of cardiac autonomic modulation during graded headup tilt by symbolic analysis of heart rate variability. Am J Physiol Heart Circ Physiol 2007;293:H702-708.

Raschi E, Vasina V, Ursino MG, Boriani G, Martoni A, De Ponti F. Anticancer drugs and cardiotoxicity: Insights and perspectives in the era of targeted therapy. Pharmacol Ther 2010;125:196-218.

Riesenberg H, Lübbe AS. In-patient rehabilitation of lung cancer patients: a prospective study. Support Care Cancer 2010;18:877-882.

Rock CL, Doyle C, Demark-Wahnefried W, Meyerhardt J, Courneya KS, Schwartz AL, Bandera EV, Hamilton KK, Grant B, McCullough M, Byers T, Gansler T. Nutrition and physical activity guidelines for cancer survivors. CA Cancer J Clin 2012;62:243-274.

Sanchis-Gomar F, Lucia A, Yvert T, Ruiz-Casado A, Pareja-Galeano H, Santos-Lozano A, Fiuza-Luces C, Garatachea N, Lippi G, Bouchard C, Berger NA. Physical inactivity and low fitness deserve more attention to alter cancer risk and prognosis. Cancer Prev Res (Phila) 2015;8:105110.

Schmitt J, Lindner N, Reuss-Borst M, Holmberg HC, Sperlich B. A 3-week multimodal intervention involving high-intensity interval training in female cancer survivors: a randomized controlled trial. Physiol Rep 2016;4. pii: e12693.

Shin HC, Yang JO, Kim SR. Effects of circuit exercise on autonomic nerve system of survivors after surgery of breast cancer. J Phys Ther Sci 2016; 28:2898-2903.

Uzunlulu M, Telci Caklili O, Oguz A. Association between metabolic syndrome and cancer. Ann Nutr Metab 2016;68:173-179.

Vainshelboim B, Müller J, Lima RM, Nead KT, Chester C, Chan K, Kokkinos $\mathrm{P}$, Myers J. Cardiorespiratory fitness, physical activity and cancer mortality in men. Prev Med 2017;100:89-94.

Vigo C, Gatzemeier W, Sala R, Malacarne M, Santoro A, Pagani M, Lucini D. Evidence of altered autonomic cardiac regulation in breast cancer survivors. J Cancer Surviv 2015;9:699-706. 